\title{
Surface Rippling \& Ion Etch Yields of Diamond Using a Focused Ion Beam: With or Without Enhanced-Chemistry, Aspect Ratio Regulates Ion Etching
}

\author{
W. MoberlyChan, T. Felter, \& M. Wall, \\ Lawrence Livermore Nationaal Lab., CMS, 7000 East Ave, Livermore, CA.
}

With a goal to FIB diamond-micromachining-toolbits for future nanomachining tools, there can be potential need to process an irregularly-shaped diamond tip (Figure 1) to a well controlled tip, and shape (Figure 1c). Two important concerns are the "speed" of FIB-ion-etch processing and also resulting surface finish. Diamond ion etches slowly, and the rate can be enhanced by more-grazing incidence angle and/or chemistryenhanced FIB etching, with water vapor providing a practical chemical etchant. The work of Adams, et al [1] provides a thorough basis for understanding the effects of angle and water on yield (\#atoms etched per ions in). Although yields can be improved a factor of 10 by control of water and angle, many geometries of FIB micromachining are limited by aspect ratio. Furthermore, chemically enhanced etching requires optimization of many other FIB processing parameters, which are otherwise considered noncritical variables in FIB processing. Water can chemically enhance FIB etching of diamond (Figures $2 \& 3$ ); however, the addition of too much water vapor diminishes the vacuum and the etch rate. Using a gas injection system (GIS needle), the vapor pressure at the sample surface may reach 10(-2) Torr, while the remainder of the vacuum system retains $5 \times 10(-5)$ Torr. Furthermore, the water-enhancement is only significantly activated if other FIB parameters such as dwell time, scan-overlap, and beam current are modified. Typically FIB current has no affect on normalized yield (\# atoms off per ion in); however, with chemical enhanced etching of diamond, the enhancement can be more for conditions of lower beam current. Thus in a less practical sense, the chemistryenhanced etching rate may only be achieved by first reducing the non-enhanced rate. For many geometries, the orientation, redeposition, and aspect ratio may limit the achieved yield enhancement (Figure 4 [2]).

Ion etching, whether FIB or other forms, has a historic bane of inducing surface roughening. Although many procedures have been developed to produce smoother surface finish (e.g. for ion-etched TEM samples), there may be utilities for controllably roughened surfaces; for example, to template self-assembly nanostructures. Ion etching at a grazing angle results in a rippled surface modification $[1,3]$, with diamond exhibiting 3 distinct regimes of ripples [1]; smoothening for angles normal $\left(0^{\circ}\right)$ up to $\sim 40^{\circ}$ incidence, ripples normal to the tilt angle for angles $40^{\circ}$ to $70^{\circ}$ (e.g. Figure 5), and step-terraces for more-grazing angles (Figure 6). When water vapor is used to chemically enhance a faster FIB etch rate, the horizontal ripples can break up into stepterrace formations at lower angles (Figure 7). Ripples are a function of ion energy and temperature, as well as angle-of-incidence, and can be disrupted by numerous FIB-scanning parameters. However, these ripples do not exhibit a significant dependence on crystallographic orientation of the single crystal diamond [4]. SEM imaging in the DualBeam (FEI NOVA600) provides instant metrology (even movies can quantify ripple growth [4]). Normal view (Figure 6a) measures wavelength; controlled tilting SEM can monitor amplitudes; and a combined SEM tilt view with FIB-induced pointers (Fig. 7) can measure etch depth independent of the precision of the FIB crater sidewalls [2]. The ripple wavelengths grow larger than typically reported [1], and this is partly a result of the higher fluence achieved in these experiments, as well as an indication of boundary conditions and aspect ratio modifying the ripples. When considering both water-enhanced and non-enhanced etching, the ripples appear more dependent on aspect ratio than on fluence (Figures 8a and 8b). However, the ripples may not enlarge in a linear fashion, especially when considering the effects of boundaries [4]. The ripples nearest the leading sidewall in Figure 5 exhibit a wavelength $150 \%$ that of the center of the etch crater.

Ref. 1. Adams, D.P., et al "FIB milling of diamond \& Effects of H2O", J. Vac. Sci. Tech. B V21(6) p2334-43, 2003.

Ref. 2. Moberlychan, W.J., et al "Effects of aspect ratio on FIB etching of diamond" in submission to JVST.

Ref. 3. Bradley, R.M. \& J.M.E. Harper, "Theory of ripple topography", J. Vac. Sci. Tech. A V6(4) p2390-95, 1988..

Ref. 4. Ichim, S. \& M.J. Aziz, "Lateral templating of self-organized ripples", J.Vac.Sci.Tech. B V23(3) p1068-71, 2005. This work was performed under the auspices of the United States Department of Energy by the University of California, Lawrence Livermore National Laboratories under contract of No. W-7405-Eng-48. 


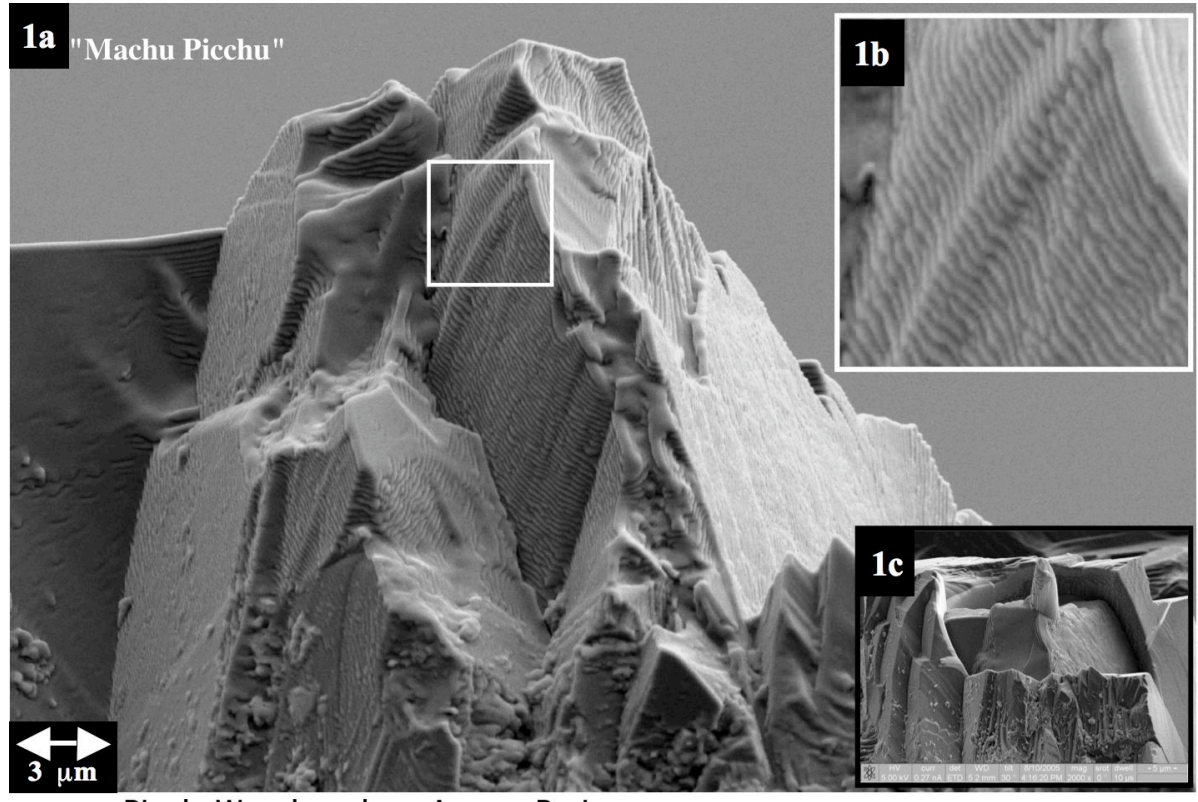

Ripple Wavelength vs Aspect Ratio
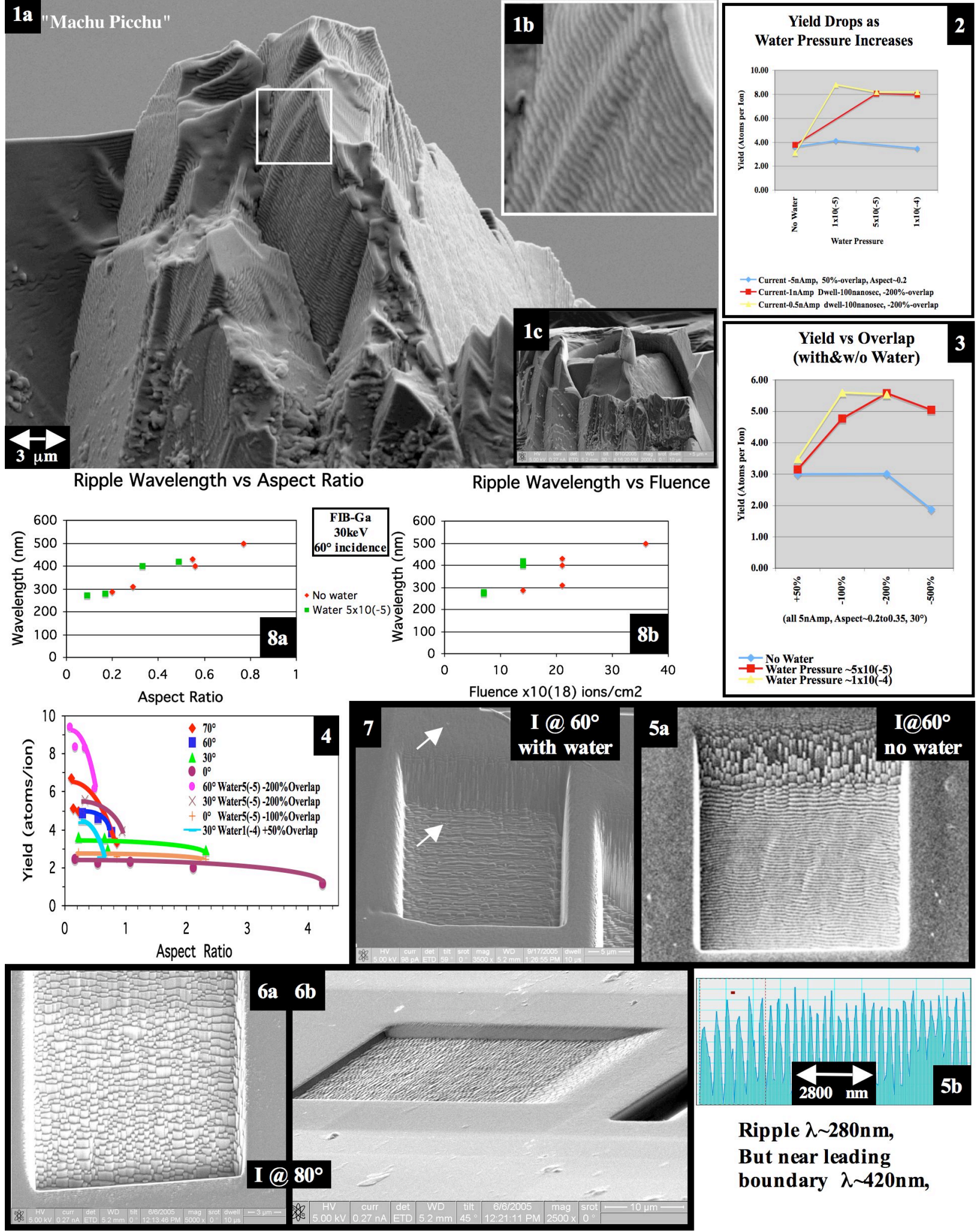

Ripple $\lambda \sim 280 \mathrm{~nm}$,

But near leading boundary $\lambda \sim 420 \mathrm{~nm}$,

FIG. 1. SEM images of an irregular diamond micromachined to a sharp tip by FIB. FIG. 2, 3, 4. Etch rate can be enhanced by grazing incidence and use of water vapor, but diminished by aspect ratio. FIG. 5, 6, 7. SEM depicts surface rippling induced by FIB at $60^{\circ}$ incidence \& step/terrace formation at higher incidence planes. FIG. 8. Ripple wavelength depends on ion energy, angle, fluence, (temperature, vapor), and also aspect ratio. 